\title{
The Bus Line Supporting System Based On Learning Neural Network Model Applied In GIS
}

\author{
Jun Song ${ }^{1, a^{*}}$, Shengwei Quan ${ }^{2, b}$ \\ ${ }^{1}$ School of Traffic and Transportation, Southwest Jiaotong University, Chengdu, 610031, China \\ ${ }^{2}$ School of Traffic and Transportation, Southwest Jiaotong University, Chengdu, 610031, China \\ aemail:841231729@qq.com , bemail:qswwood@163.com
}

Keywords: Bus routes selection artificial neural network; Traffic congestion distribution; GIS

\begin{abstract}
With the rapid increase of urban population and rapidly widening scope of the city, the newly built residential areas emerging or leading to the existing bus lines could not meet the needs of the people's activities. The phenomenon of the traffic congestion is becoming more and more serious. [1] According to the problem we based on the principle of convenient resident travel, we used the point - line - face integration optimization model to slow down the urban traffic congestion as the goal, combined with the population distribution model and the congestion nodes judging model. We make the city Chengdu as the research object and after our research the Chengdu bus route selection model is established. [2]At the same time, on the basis of this model combining with the ArcGIS technology, database technology achieved in the host framework programming language $\mathrm{C} \#$ helped with the tool MATLAB combined with $\mathrm{C}++$ language dynamic link library files and the algorithm model developed by the bus route selection model, we achieved our information system according the message or rules we mentioned in this passage. We compared our city bus route selection in the last part which we designed to the original route scheme optimization selection and further demonstrate the superiority of the new scheme. [3]
\end{abstract}

\section{Introduction}

The dramatic increase of population and the residential expansion in urban area will inevitably call larger and larger demand of the transportation, and it will result in traffic congestion. Factors caused by traffic jams are surrounding with the imbalance of the road population distributions, the capacity of road network and the traffic flow of roads each peak hour. That means it will increase the number of traffic flow translational velocity, the amount of motor vehicles, the number of traffic lights, etc. Based on the problem mentioned above in this passage, we catch the thought of the space factors and the traffic congestions in space appeared with three kinds of states like point, line and plane:

Point - It refers to the intersection of traffic congestion occurs separately which is only connected to its roads, and will not affect the adjacent intersections.

Line - It refers the traffic flow line corresponds with points due to some issues such as peak flows or the local traffic congestion which was not timely solved.

Plane - It refers to the traffic congestion related with sections overlapping between the road and road intersection and all of these points and lines constitute an appropriately traffic congestion.

The model assumptions are listed below:

The present study to optimize the bus line around the scope of population distribution in line follows a pattern that the nearly population increase gradually as the instance increasing. We assume that the population distribution is roughly accord with normal distribution along the bus lines.

We assume that the daily average traffic flow per hour are roughly equal besides 7:00-9:00 and 18:00-20:00 when there is no emergency situation and the choice for route area within the scope of the traffic is equal to drivers.

It's equal to drivers for all choices of the route. 


\section{Symbols:}

TCRI $_{\text {link }}$

$N_{i}(k)$

The 'Jam' weighted coefficient

T----- Test cycle

$C_{i}$---- The Ith Lane capacity

$T C T I_{\text {road }}$----- 'Jam' weighted coefficient (How greater the value is means how serious the congestion

is.)

$\bar{v}_{j i}(k)$---- The counting average vehicle speed in the Kth period with section $\mathrm{J}$ in I

$\overline{v_{j o}}(k)$---- The maximum speed of the Kth cycle in allowed section J set

\section{Traffic distribution model of point, line and plane}

\section{Congestion point -- weighted coefficient of road traffic congestion in section}

The judgment of 'congestion point' is to collect information about road congestion from the traffic information system, and then get the congestion place. Generally speaking, we take the road traffic capacity $\mathrm{V}$ and road traffic flow $\mathrm{C}$ ratio to determine the condition of the traffic flow, so as to meet the weighted coefficient of the determination model for 'congestion point'.

$$
T C R I_{\text {link }}=\frac{\sum_{i=1}^{n} \frac{N_{i}(k)}{T}}{\sum_{i=1}^{n} C i}
$$

\section{Congestion line -- weighted coefficient of road traffic congestion in line}

Congestion line - line congestion weighted coefficient 'Congestion line' gets the information whether congestion point in the road is linked together. If there is no coincidence, it means 'congestion point' is relative in dependence of the traffic jam, and they can be solved more intuitively and conveniently. If the beginning and the end of the road have overlapped, it means that sections are linked together. They constitute a 'congestion line'. The 'congestion line' weighted coefficient for the model (In table1, we list the ranges of the $T C R I_{\text {link }}$ and $T C R I_{\text {road }}$.):

$$
\text { TCRI }_{\text {road }}=\frac{v_{j o}(k)}{\frac{1}{n} \sum_{i=0}^{n} v_{j i} \overline{(}(k)}-1
$$

\begin{tabular}{|c|c|c|c|c|c|}
\hline \multirow{2}{*}{$\begin{array}{c}\text { Class } \\
\text { definition }\end{array}$} & Level 1 & Level 2 & Level 3 & Level 4 & Level 5 \\
\hline & Very smooth & Smooth & $\begin{array}{c}\text { Slight } \\
\text { congestion }\end{array}$ & $\begin{array}{c}\text { Intermediate } \\
\text { congestion }\end{array}$ & $\begin{array}{c}\text { Severe } \\
\text { congestion }\end{array}$ \\
\hline $\begin{array}{c}T C R I_{\text {link }} \\
\text { range }\end{array}$ & {$[0,2]$} & {$[2,4]$} & {$[4,6]$} & {$[6,8]$} & {$[8,10]$} \\
\hline $\begin{array}{c}T C R I_{\text {road }} \\
\text { range }\end{array}$ & {$[0,2]$} & {$[2,4]$} & {$[4,6]$} & {$[6,8]$} & {$[8,10]$} \\
\hline
\end{tabular}

Table 1 'congestion point', 'congestion line' congestion coefficient defined criteria range 


\section{The development of Congestion nodes domain traffic flow which spreads in space distribution with the duration.}

Firstly, we use the method of correlation analysis and fuzzy cluster analysis to study the traffic congestion's change spreads with the development of the time and space.

To get the information of the development trend about traffic congestion in space, we need to consider the change of the traffic flow with its associations. And we can use the method of statistical correlation analysis to determine through the historical data of traffic flow.

$$
r=\frac{\sum_{i=1}^{n}\left(x_{i}-\bar{x}\right)\left(y_{i}-\bar{y}\right)}{\sqrt{\sum_{i=1}^{n}\left|x_{i}-\bar{x}\right|^{2} \sum_{i=1}^{n}\left|y_{i}-y\right|^{2}}}
$$

The $r$ is called correlation coefficient and it has three properties:

(1) The absolute value of $r$ is less than or equal to $1,|r| \leqslant 1$.

(2) There exists strict linear relationship between that $y i=a+b * x i, i=1,2,3, \ldots n$. to have $|r|=$ 1 , and when $\mathrm{b}>0, \mathrm{r}=1 ; \mathrm{b}<0, \mathrm{r}=1$.

(3) If there exists no relationship between $\mathrm{x}$ and $\mathrm{y}, \mathrm{r}$ equals to 0 on average.

We notice that there is a time lag when a traffic flow comes from one section to another section. Therefore, at the time of getting samples, we consider involve two sections in pair as samples. Such as $\mathrm{Ai}$ and $\mathrm{Bi}$ and we take the value of time as the value of $\mathrm{At}$, and regard the value as $\mathrm{Bt}+\mathrm{k}$ at the $\mathrm{t}+$ $\mathrm{k}$ time, the value $\mathrm{k}$ means the difference that how long the traffic flow goes between $\mathrm{A}$ and $\mathrm{B}$ section. So, it is able to judge the correlation of the traffic flow from the correlation coefficient.

After calculating the road correlation, we can get the road network traffic congestion evaluation according to the theory of fuzzy clustering which is in analysis of all the sections of road network clustering. And we use fuzzy mathematics to compute the similarity relationship after considering a fact that the correlation in road traffic is not very clear and it is fuzzy.

According to the similarity coefficient method mentioned above in this passage, we got a matrix named $\mathrm{R}$ and this matrix can be directly used in the fuzzy clustering analysis which is called fuzzy similar matrix in fuzzy mathematics.

At this time, we can modify and reconstruct $\mathrm{R}$ matrix using the existing exponentiation relationship with $\mathrm{R}$ and its correlation variables. We can also get the answer of the transitive closure of $R$ with the flat method $t(R)$, and we can comprehend that the transitive closure is a fuzzy equivalence matrix for something where you can get the clustering figure at an appropriate level after it is taken on lambda. According to the lambda, we make an assumption that we have already calculated the shortest path midpoint set and we can also obtain them with different length of paths to weight sorting. At the same time, in the weight multiplied by the basis of all congestion coefficient and population distribution coefficient (congestion coefficient and the surrounding with the population distribution coefficient of road congestion coefficient and congestion line includes congestion points corresponding sections of the corresponding with the congestion route) [4] ,it can be obtained after different path curvature of the population distribution and we will realize the blockage of weighted value.

After the confirmation of the algorithm mentioned above in this passage, we use our model in map mode generated by ArcGIS and input the grid data entry at the same time. [5] After these preparations, we also reconstruct our model code in the information system based on the previous code considering the traffic congestion coefficient under the electronic maps in the basis of the reality of scheme generation bus route.

\section{New design route optimization:}

After making the better establishment of the route from our school to Chengdu railway station, we compared the result with the original route scheme. In the figure1 and figure 2 below in this passage, 
we test the software whether owns the ability to automatically avoid the congestion coefficient 'jam' and the 'congestion line' as much as possible based on entry parts of geographic coordinate point congestion values existing in the background database

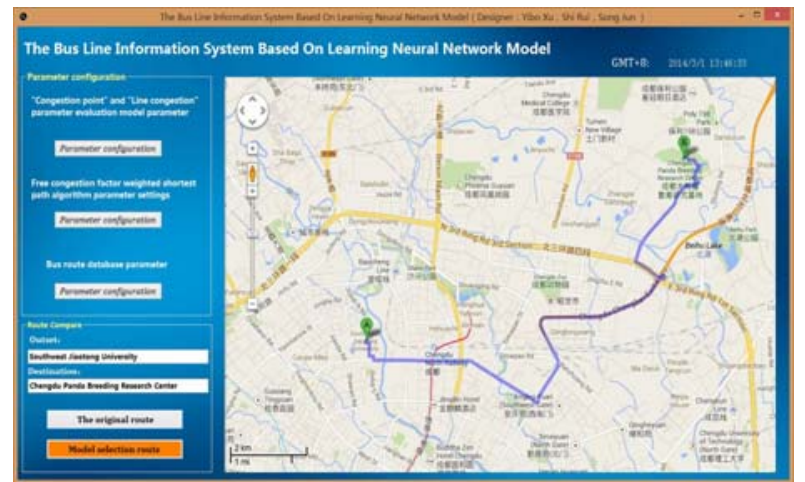

Fig. 1 New bus route scheme (congestion weighted factors)

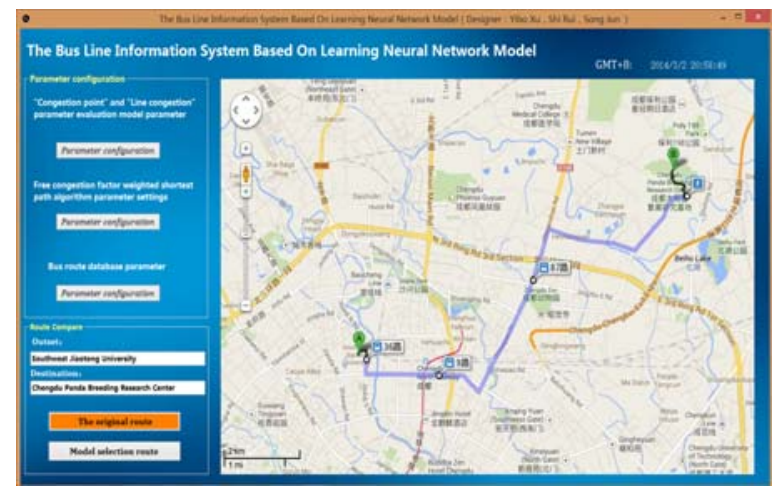

Fig. 2 The original line of the new route selection scheme Graphic description: The original plan which appears in black line style in maps; The plan after considering congestion factors appears in red line style in maps

\section{Conclusion}

Through our test and comparison, the system of the newly generated route to avoid congestion coefficient larger 'jam'. The 'congestion line' so as to avoid the congestion degree higher 'jam' is more humanly, efficiently and conveniently. In the reality of the electronic map, for the reason that ArcGIS doesn't support the population's distribution in secondary development use, the author import the substitute e in population distribution model based on the reality of electronic map Therefore, the model has higher bus route selection value and it's more promotable in space.

\section{References}

[1] Jun Song, The City Traffic Service Platform Based On MPI Model, Applied Mechanics and Materials, v548-549, p 1844-1847, 2014, Achievements in Engineering Sciences. (2014)

[2] Xingwei Liu, Traffic Management Staff System Based On MARKOV Modal, Applied Mechanics and Materials, v556-562, p 4582-4585, 2014, Mechatronics Engineering, Computing and Informaion Technology. (2014)

[3] Fang $\mathrm{Xu}$, ANALYSIS OF THE MODEL OF COMMUNITY SPORTS LOGISTICS DISTRIBUTION CENTER LOCATION UNDER ELECTRONIC COMMERCE, ICTE2013. (2013)

[4] Jingfei Li, Wavelet technology and network traffic prediction based on ARIMA models, Shandong University. (2010)

[5] Jiaojiao Li, Chongchang Wang, ArcGIS-based CAD to GIS data conversion, Technology \& Economy. (2011) 